\title{
Genetica
}

December 2014, Volume 142 Issue 6 Pages 545-554

http://dx.doi.org/10.1007/s10709-014-9802-5

http://archimer.ifremer.fr/doc/00247/35811/

(c) Springer International Publishing Switzerland 2014

\section{Cytogenetic evidences of genome rearrangement and differential epigenetic chromatin modification in the sea lamprey (Petromyzon marinus)}

\author{
Covelo-Soto Lara ${ }^{1,{ }^{*}}$, Moran Paloma ${ }^{1}$, Pasantes Juan J. ${ }^{1}$, Perez Garcia Maria Concepcion ${ }^{1,2}$
}

${ }^{1}$ Univ Vigo, Dept Bioquim Xenet \& Inmunol, Vigo 36310, Spain.

${ }^{2}$ IFREMER, Dept Biogeochem \& Ecotoxicol, Lab Ecotoxicol, F-44311 Nantes 03, France.

* Corresponding author : Lara Covelo-Soto, email address : laracovelo@uvigo.es

\begin{abstract}
:
This work explores both the chromatin loss and the differential genome methylation in the sea lamprey (Petromyzon marinus) from a molecular cytogenetic point of view. Fluorescent in situ hybridization experiments on meiotic bivalents and mitotic chromosomes corroborate the chromatin loss previously observed during the development of the sea lamprey and demonstrate that the elimination affects not only to Germ 1 sequences but also to the rpt200 satellite DNA and most part of the major ribosomal DNA present on the germinal line. 5-Methylcytosine immunolocation revealed that the GC-rich heterochromatin is highly methylated in the germ line but significantly less in somatic chromosomes. These findings not only support previous observations about genome rearrangements but also give new information about epigenetic changes in P. marinus. The key position of lampreys in the vertebrate phylogenetic tree makes them an interesting taxon to provide relevant information about genome evolution in vertebrates.
\end{abstract}

Keywords : Sea lamprey, Chromatin loss, Genome rearrangements, DNA methylation 
Lampreys (Agnatha, Petromyzontiformes) are one of the major extant lineages of jawless vertebrates (Takezaki et al. 2003). These organisms are crucial in the understanding of early vertebrate evolution because they constitute, together with hagfishes, the most primitive group of living vertebrates and represent the vertebrate ancestral lineage.

Despite its key position at the vertebrate phylogenetic tree, knowledge on the genome organization of lampreys is limited. Chromosome numbers and karyotypes of approximately half of the Petromyzontidae species were described before the 80's (reviewed by Potter and Robinson 1981). With the exception of cytogenetic reports performed in the genus Lethenteron (Suzuki et al. 1999), in Lampetra zanandreai (Caputo et al. 2011) and in Petromyzon marinus (Smith et al. 2010), no new cytogenetic data was published since then. The karyotypes of lamprey species are usually characterized by high numbers of small sized chromosomes ( $2 n=60$ to 180). These features, together with the difficulties in obtaining an appropriate number of well spread metaphase plates, have hindered cytogenetic studies. species of the North Atlantic and the Mediterranean. Sea lampreys spend most of their life as filter-feeding larvae that undergo metamorphosis to become parasitic adults depending on marine hosts. A diploid chromosome number of $2 n=168$ and a karyotype mainly composed of acrocentric and a few metacentric chromosomes were described (Potter and Rothwell 1970). Moreover, a satellite DNA family (EcoRI satDNA) has been located at centromeric and paracentromeric positions of several chromosome pairs (Boán et al. 1996). The accomplishment of the first sea lamprey whole-genome sequence assembly (Smith et al. 2013) gave new information about the structure and composition of the genome of $P$. marinus showing a high content of repetitive elements and a richness in GC higher than in other vertebrates (Smith et al. 2013).

On the other hand, the programmed loss of about $500 \mathrm{Mb}$ of DNA was described in several somatic lines during embryonic development of this species (Smith et al. 2009). One of the sequences partially eliminated, Germ1, consists of a 5' end somatically rare region, a fragment of the $28 \mathrm{~S}$ rDNA, the repetitive element rpt200, and the whole 18S rDNA (Smith et al. 2009). The elimination processes involve complex, tightly regulated gene networks (Chen 2007) that are, therefore, susceptible of epigenetic control through different modification mechanisms among which DNA methylation is the best known (Reik et al. 2001; Feng et al. 2010). Although changes in DNA methylation levels have been extensively studied in mammals and plants (Feng et al. 2010) 
and methylation has been recently identified as a marker of DNA elimination in the ciliate Oxytricha trifallax (Bracht et al. 2012; Bracht 2014), the characterization of the DNA methylation patterns in other organisms, including fishes is an area about which few reports have been published.

In order to determine if the programmed chromatin loss in P. marinus is related with changes in DNA methylation, we compared the chromosomal methylation levels on germinal and somatic cell lines of the sea lamprey through the immunolocation of 5-methylcytosine on gonadic meiotic bivalents and lymphocyte chromosomes. For a better understanding of the genome organization in sea lampreys and to study the chromosomal rearrangements involved in the DNA loss, we also analyzed mitotic and meiotic chromosomes of the sea lamprey by fluorochrome staining and Cbanding and compared the fluorescent in situ hybridization (FISH) signal patterns obtained after using Germ1, rpt200, EcoRI satDNA and 28S rDNA sequences as probes.

\section{Materials and Methods}

\section{Biological material}

Eight specimens of lampreys were fished in the Miño River (NW Spain). Once in the lab, blood was extracted from their caudal arteries and collected in tubes containing lithium heparin (Sigma). After euthanizing the lampreys with MS-222 (Sigma), samples of muscular and gonadal tissues were excised from each individual and conserved in absolute ethanol. Part of the gonadal tissue was also dissected in order to obtain meiotic chromosome preparations. The experimental procedure was performed with the approval of the Ethics Committee of the University of Vigo, complying with the current laws of Spain. All institutional and national guidelines for the care and use of laboratory animals were followed.

DNA extraction, Polymerase chain reaction (PCR) amplification and probe labeling

Total DNA was extracted from ethanol preserved muscular and gonadal tissues using an extraction kit (Nucleospin ${ }^{\oplus}$ Tissue, Macherey-Nagel) following the supplier instructions. FISH probes were obtained by PCR. Amplifications were performed in $20 \mu \mathrm{l}$ of a solution containing $50 \mathrm{ng}$ DNA, 1x PCR buffer, $0.5 \mathrm{mM}$ each dNTP, $2.5 \mathrm{mM} \mathrm{MgCl}, 1 \mu \mathrm{M}$ each primer and 1 U BIOTAQ DNA polymerase (Bioline). The combinations of primers used in the amplification experiments appear in Table 1. Universal primers (Vilgalys 2005) were used to amplify a fragment 
of the $28 \mathrm{~S}$ rRNA gene of the major rDNA repeat. The amplifications of the rpt200 element and the EcoRI satellite were performed using primers described in Smith et al. (2009) and Boán et al. (1996), respectively. Amplifications were realized in a GeneAmp PCR system 9700 (Applied Biosystem) using the conditions shown in Table 2. All PCR products were examined by electrophoresis on $2 \%$ agarose gels.

In order to detect exclusively the 5' end of the "somatic rare region of Germ1" (Smith et al. 2009), specific primers were designed using Primer3 v.0.4.0 designing tool (Rozen and Skaletsky 2000). The primers (Table 1) allowed amplifying the $5^{\prime}$ end of Germ1, the part of the sequence devoid of rpt200, 28S rDNA and 18S rDNA.

Germ1 and rpt200 amplifications were confirmed by cloning in a pGEM-T vector System (Promega) and sequencing using a dRhodamine terminator cycle sequencing kit (Applied Biosystems) and an automated sequencer ABI PRISM 310. $28 \mathrm{~S}$ rDNA was labeled with biotin 16-UTP by nick translation. rpt200, 5' Germ1 and EcoRI satDNA were directly labeled by PCR either with biotin-16-UTP or digoxigenin-11-UTP. The labeled PCR products were precipitated before FISH.

\section{Chromosome preparation}

Meiotic prophase I bivalents were obtained from male individuals showing well developed gonads. Fragments of tissue excised from the gonads were immersed in a hypotonic solution containing $0.05 \%$ colchicine in $0.4 \% \mathrm{KCl}$ for $1 \mathrm{~h}$. The tissue was then fixed with a solution of ethanol and acetic acid (3:1) for $1 \mathrm{~h}$. Chromosome spreads were prepared by dissociating small pieces of tissue in $60 \%$ acetic acid and dropping the cellular suspension onto clean slides heated to $58 \stackrel{\circ}{ } \mathrm{C}$.

Mitotic chromosome preparation from lymphocyte cultures followed the protocol described by Fujiwara et al. (2001) with slight modifications. Two $\mathrm{ml}$ of heparinized blood were mixed with $5 \mathrm{ml}$ of culture medium (Iscove, Sigma) containing $12 \%$ fetal bovine serum (Sigma) and $1 \%$ of an Lglutamine, penicillin and streptomycin solution (Sigma). The mixture was centrifuged at $1200 \mathrm{rpm}$ for $10 \mathrm{~min}$. The upper layer, corresponding to lymphocytes, was collected and added to $5 \mathrm{ml}$ of the supplemented culture medium. After lymphocyte stimulation with a mixture of phytohemagglutinin (167 $\mathrm{mg} / \mathrm{ml}$, Sigma) and lipopolysaccharide $(1.67 \mu \mathrm{g} / \mathrm{ml}$, Sigma), cells were cultured for six days at room temperature. 5-Bromo-2'-deoxyuridine $(20 \mu \mathrm{g} / \mathrm{ml})$ and colchicine $(0.2 \mu \mathrm{g} / \mathrm{ml})$ were added to the cultures 8 and $3 \mathrm{~h}$, respectively, before harvesting. After centrifugation at $1200 \mathrm{rpm}$ for $10 \mathrm{~min}$, cells were treated with $0.075 \mathrm{M} \mathrm{KCl}$ for 20 min at room 
temperature and fixed with a mixture of ethanol and acetic acid (3:1). Chromosome slides were obtained by dropping the cellular suspension on clean slides and air drying.

Fluorochrome staining and C-banding

Some preparations were stained for $2 \mathrm{~h}$ with CMA $(0.25 \mathrm{mg} / \mathrm{ml})$ and counterstained with DAPI $(0.14 \mu \mathrm{g} / \mathrm{ml})$ for $8 \mathrm{~min}$. Once washed with distilled water, slides were air-dried and mounted with antifade (Vectashield, Vector). After visualization and photography, preparations were washed and re-stained with a combination of DAPI $(0.14 \mu \mathrm{g} / \mathrm{ml})$ and PI $(0.07 \mu \mathrm{g} / \mathrm{ml})$. The slides were then washed in distilled water, air-dried, mounted in antifade and photographed again. C-banding was performed following the technique of Sumner (1972). The chromosomes were stained with acridine orange $(0.05 \mathrm{mg} / \mathrm{ml})$ instead of Giemsa as proposed by Bella et al. (1986).

Immunolocation of 5-methylcytosine (5-mC)

Methylated cytosines were detected using a mouse antibody against 5-mC (Eurogentec) following the protocol indicated by the supplier with minor modifications. Mitotic and meiotic chromosome preparations were treated with a blocking solution (1\% bovine serum albumin (BSA) in phosphate saline solution Tween-20 (1xPBST)) for $30 \mathrm{~min}$ and then incubated with the 5-mC antibody for 90 $\min$ at $37^{\circ}$ C. After washing in blocking solution, the 5-mC antibody was detected with a FITC (fluorescein isothiocyanate)-conjugated goat anti-mouse secondary antibody (Sigma).

Fluorescent in situ hybridization (FISH)

Single and double FISH experiments followed the methods published by Pérez-García et al. (2010) with minor modifications. Preparations were denatured at 69 ㅇ $\mathrm{C}$ for $2 \mathrm{~min}$ and hybridized overnight at 37 으. Stringency washes were performed with $50 \%$ formamide in 2xSSC (saline-

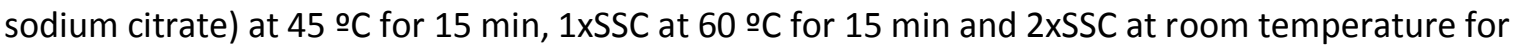
$5 \mathrm{~min}$.

164 Signal detection was carried out with fluorescein avidin and biotinylated anti-avidin (Atom) for the biotinylated probes and with mouse anti-digoxigenin, goat anti-mouse TRITC

166 (Tetramethylrhodamine isothiocyanate) and rabbit anti-goat TRITC (Sigma) for the digoxigenin167 labeled probes. Slides were counterstained with DAPI or DAPI/PI and mounted in antifade. 
Slide visualization and photography were performed with a Nikon Eclipse-800 microscope equipped with an epifluorescence system. Separated images for each fluorochrome were obtained with a DS-Qi1Mc CCD camera (Nikon) controlled by the Nis-elements software (Nikon). Merging of the images was performed with Adobe Photoshop. Chromosome counting was performed using the Nis-elements software (Nikon).

\section{Statistical evaluation}

In order to compare the degree of methylation of the GC-rich bands in meiotic and mitotic cells, we determined the number of $5-\mathrm{mC}$ antibody in 32 mitotic metaphase and 32 meiotic prophase I plates (Table 1 in Supplementary Files). The numbers of EcoRI satDNA, rpt200 and 28S rDNA FISH signals were also determined in the same cells. In order to assess the possible reduction of these genome marks, the numbers of the mitotic signals were compared with the number of expected signals, calculated as the double of the meiotic signals, applying a Wilcoxon signed-rank test using an IBM software (IBM corp. Released 2010. IBM SPSS Statistics for Windows, Version 19.0).

\section{Results}

Fluorochrome staining and C-banding

Mitotic metaphase chromosome analysis of all specimens of $P$. marinus shows a diploid complement of $2 n=168$ chromosomes whereas meiotic prophase I plates present a haploid complement of $n=99$ chromosomes (Fig. 1). Combined DAPI/CMA staining of mitotic chromosomes and meiotic bivalents reveals CMA bright bands at one of the terminal regions of every meiotic bivalent and mitotic chromosome (Fig. 1a, b, g, h). These CMA positive regions are also DAPI negative (Fig. 1C, i), coincident with C-positive bright bands (Fig. 1d, j) and showing also bright fluorescence after PI staining (Fig. 1e, k). DAPI staining also reveals a banding pattern on the chromosomes; while some of the mitotic chromosomes are poorly or intensely stained with DAPI, others show alternate dull and bright regions. These differences are clearer on meiotic plates in which most bivalents show a pattern of alternate dull and bright DAPI bands (Fig. 2a, c). Some of these bright DAPI bands are coincident with faint C-positive bands (Fig. 1d, j).

Immunolocation of 5-methylcytosine 
202 The methylation patterns of the chromosomes of the sea lamprey P. marinus were analyzed on

203200 mitotic metaphase and meiotic prophase I plates from eight individuals. Strong 5-mC signals

204 appear on terminal regions of all bivalents on meiotic plates. These signals are coincident with the

$205 \mathrm{CMA}+$ /PI+/DAPI- regions (Fig. 2b, some signaled by arrows). Intercalary signals were also present

206 on some regions showing dim DAPI fluorescence (Fig. 2a, b, arrowheads). 5-mC signals are also

207 located on the terminal CMA+/PI+/DAPI- regions of the mitotic chromosomes (Fig. 2c, d, arrows)

208 but they are fainter and not present in every single chromosome of the mitotic plate. No signals

209 were detected in at least 20 mitotic chromosome pairs (Fig. 2c, d, arrowheads). After carefully

210 counting the number of positive signals in 32 meiotic and 32 mitotic plates (Table 3 ), the

211 application of the Wilcoxon signed-rank test to the data showed that the number of mitotic 5-mC

212 signals shows a significant reduction $(Z=-4.938, p<0.001)$ with respect to the number of signals

213 expected, that is the double of the number detected in meiotic plates counts.

215 Fluorescent in situ hybridization

217 FISH experiments using a biotin labeled EcoRI satDNA probe were performed in both meiotic

218 bivalents and mitotic chromosomes from 8 specimens of $P$. marinus. Analysis of 200 chromosome

219 plates showed signals on 17 bivalents and 31-34 mitotic chromosomes (Fig. 1e, k). The detection

220 of all 34 signals in a single mitotic metaphase plate was difficult, mostly due to the small size of

221 some chromosomes and its high number. EcoRI satDNA signals appear on bright DAPI bands, close

222 to the CMA/PI positive regions (Fig. 1e, k). In five of the DAPI-bright bivalents and mitotic

223 chromosome pairs, the signals cover most part of the chromosome (Fig. 1c, e, i, k, arrowheads)

224 while in others the size of the signal is smaller (Fig. 1e, f, arrows). In mitotic chromosomes some

225 of these small signals seem to overlap the CMA positive regions (Fig. 1k, I, arrows). The numbers

226 of FISH signals detected in 32 meiotic prophase I and 32 mitotic metaphase plates appear

227 resumed in Table 3. In contrast with the reduction of the 5-mC signals, the Wilcoxon signed-rank

228 test revealed that the number of EcoRI satDNA FISH signals is not significantly different $(\mathrm{Z}=-1.738$,

$229 \mathrm{p}=0.082$ ) from the double of those detected in meiotic plates.

230 The repetitive element rpt200 was mapped by FISH with a digoxigenin labeled probe. Meiotic

231 prophase I plates show signals on DAPI-dull regions of 20 bivalents. Most signals are close to the

232 bright CMA/PI regions (Fig. 1f, asterisks). In contrast, only 20 to 30 mitotic chromosomes, and not

233 the expected 40, present rpt200 signals. Most of these signals seem to overlap the CMA/PI

234 positive regions on mitotic chromosomes (Fig. 1l, asterisks). Table 3 shows the number of rpt200

235 FISH signals in 32 meiotic prophase I and 32 mitotic metaphase plates. The number of rpt200 FISH 
236 signals is significantly lower $(Z=-4.963, p<0.001)$ than expected taking into account the number

237 of signals detected in meiotic plates.

238 Double FISH experiments demonstrated that signals for EcoRI satDNA and rpt200 map to different

239 meiotic bivalents and chromosome pairs (Fig. 1f, I). In the case of EcoRI satDNA, posterior C-

240 banding of the same plates confirmed that some signals are located on C positive bands on

241 meiotic bivalents and on mitotic chromosomes (Fig. 1d, j).

242 Major ribosomal genes were mapped by FISH using a biotin labeled fragment of the coding region

243 of the $28 \mathrm{~S}$ rDNA (Fig. 3a, c, d, f). This $28 \mathrm{~S}$ rDNA probe covers part of the $28 \mathrm{~S}$ rDNA segment

244 included in the Germ1 sequence. FISH signals were detected in 9 bivalents (Fig. 3a). In contrast, a

245 single location in one chromosome pair was present in mitotic metaphase plates (Fig. 3d). Double

246 FISH experiments using 28 S rDNA and rpt200 probes labeled differently revealed that all $28 \mathrm{~S}$ rDNA

247 signals coincide with rpt200 signals, but there are some rpt200 signals devoid of $28 \mathrm{~S}$ rDNA signals,

248 too (Fig. 3C, f). The 5' end somatically rare region of Germ1 probe was used to determine the true

249 localization of the Germ1 sequence with respect to the $28 \mathrm{~S}$ rDNA position (Fig. 3g, h, j, k). Double

250 FISH experiments revealed that 5' end somatically rare region of Germ1 and 28S rDNA signals co-

251 locate in both meiotic and mitotic chromosomes (Fig. 3i, I). A schematic representation of the

252 main types of meiotic and mitotic chromosomes, attending to their cytogenetic markers, is shown

253 in the Figure 4.

\section{Discussion}

Programmed loss of chromatin, far from an unusual event, seems to be a common process of cell differentiation and development. Chromatin elimination has been reported in somatic cell lines during embryonic development of nematodes, copepods, insects, marsupials, hagfish (reviewed by Kloc and Zagrodzinska (2001)) and in the sea lamprey P. marinus (Smith et al. 2009; Smith et al. 2010). As previously described by Smith et al. (2010), discrepancies in haploid complement were detected between lymphocytes $(n=84)$ and germ-line cells $(n=99)$, indicating that chromosomal fusion and/or chromosome loss occurred during development. The lymphocyte chromosome counts obtained in this work $(2 n=168)$ coincide with the numbers previously described by Potter and Rothwell (1970), but differs with respect to the diploid chromosome number $(2 n=164)$ reported by Smith et al. (2010). Given the small size of the sample and the difficulties in accurately counting large numbers of small chromosomes, the differences might be due to variation among individuals but also to a loss of chromosomes during the spreading caused by strong hypotonic treatment. 
The FISH experiments presented here corroborate the Germ1, 28S rDNA and rpt200 loss observed by Smith et al. (2009) in the somatic line but also show that rpt200 appears in locations devoid of Germ1, confirming that rpt200 is not exclusively a part of the Germ1 sequence. Taking into account that Germ1 and major rDNA signals co-localize with rpt200 signals in meiotic bivalents (Fig. 4), most of the rpt200 signal loss squares with major rDNA and Germ1 elimination (Smith et al. 2009). The rpt200 signals still present in the somatic line mostly correspond to rpt200 loci not associated to Germ1 with the exception of the single remaining nucleolar organizer region of mitotic chromosomes. Chromatin loss has been associated to elimination of heterochromatic regions in hagfishes, copepods and nematodes (Nakai et al. 1991; Müller and Tobler 2000; Grishanin 2014). Nonetheless, the terminal heterochromatic regions detected in all meiotic bivalents of $P$. marinus are still present in somatic mitotic chromosomes. The presence of these GC rich heterochromatic regions is consistent with the high GC content of the genome of $P$. marinus (Smith et al. 2013). Moreover, we also detected significant differences in the degree of methylation of germ and somatic cell lines. DNA methylation has been associated with the structural maintenance of the genome (Matzke et al. 2000; Jones 2012) and the control of repetitive elements (Suzuki and Bird 2008; Feng et al. 2010). Some of the DNA methylation signals detected could be involved in those processes, but the significant reduction in their number probably indicates that most of the signals are related with the control of the chromatin loss. In this sense, methylation has been recently identified as a marker of DNA elimination in the ciliate Oxytricha trifallax (Bracht et al. 2012; Bracht 2014). Although the reduction in the 5-mC signals could also be partly attributed to technical problems associated to the differences in chromosome compaction of meiotic bivalents and mitotic chromosomes, the absence of differences in the number of EcoRI satDNA FISH signals in the same cells indicates that it is not the case. Moreover, specific cell-type DNA methylation patterns due to regulatory landscape have been described in some vertebrates (Laurent et al. 2014). However, most of the DNA methylation status remains unchanged in all tissues and only a small portion is considered tissue-specific (Dricu 2012). Nevertheless, further studies are necessary to determine the implication of these processes on the methylation differences detected in lampreys.

299 Although the presence of bright CMA bands at terminal positions on the chromosomes of $P$.

300 marinus points to the presence of GC-rich DNA satellites in this species, the only satellites thus far 301 characterized in lampreys, EcoRI and rpt200, are relatively rich in AT. Both satellites map to 302 different chromosome pairs and the signals are contiguous to the terminal CMA+/DAPI-/C- bands in meiotic prophases, but on mitotic chromosomes seem to overlap these regions. These 
apparent overlapping detected in mitotic chromosomes can be attributed to limitations in the resolution power of the technique partly due to the high condensation degree of these chromosomes. EcoRI satDNA signals always appear on DAPI+ regions, as expected from a $59.19 \%$ AT-richness. On the other hand, rpt200 signals map to chromosomal regions without differential DAPI staining despite their richness in AT (54.68\%). The hybridization results obtained for EcoRI satDNA are in accordance with those provided by Boán et al. (1996) but clearly differ from those found in Lampetra zanandreai, in which the EcoRI satDNA only appears at the centromeric region of a single chromosome pair (Caputo et al. 2011). In contrast with most other eukaryote species, the heterochromatin detected by $\mathrm{C}$-banding in P. marinus appear on both AT-rich (CMA $/ \mathrm{DAPI}+$ ), and GC-rich (CMA+/DAPI-) regions. Thus far, GC-rich heterochromatin have only been previously reported in the cyprinid Gobio gobio (Kirtiklis et al. 2005), in several other fish species of the genus Chromaphyosemion (Völker et al. 2005) and in the lamprey Lampetra zanandreai (Caputo et al. 2011). As also happens in the fish Piaractus mesopotamicus (Almeida-Toledo et al. 1998), the bright signals obtained after 5-methylcytosine immunolocation are located in the GC-rich heterochromatic regions, demonstrating that on meiotic chromosomes these regions are highly methylated and, therefore, transcriptionally repressed (Jaenisch and Bird 2003). The phylogenetic relationships between lampreys and the rest of the vertebrate groups and the deepness of their lineage ancestry, makes genome characterization of lampreys an interesting subject that might provide relevant information about genome evolution in vertebrates. Here we confirmed the chromatin loss in the somatic line and demonstrated that the elimination affects not only to Germ1 sequences but also to the rpt200 satellite DNA and most part of the major ribosomal DNA present on the germinal line. We also found that the GC-rich heterochromatin regions of germ and somatic lines show different methylation patterns. The change in the methylation pattern, its GC-rich heterochromatin and the elimination of chromatin during its development show that lampreys are an intriguing taxonomic group in the tree life meriting further research.

333 We wish to thank Pilar Alvariño for her technical assistance and Manuel Ángel Pombal Diego and

334 Manuel Megías Pacheco for kindly providing the lampreys. This work was partly funded by grants 335 from Xunta de Galicia and Fondos FEDER: "Unha maneira de facer Europa" 336 (PGIDIT03PXIC30102PN; 08MMA023310PR; Grupos de Referencia Competitiva, 2010/80; Grupos 
con Potencial Crecimiento, GPC2013-011). The author Lara Covelo-Soto has a predoctoral fellowship from the Xunta de Galicia (Plan 12C).

\section{Conflict of interest}

The authors declare that they have no conflict of interest.

\section{References}

Almeida-Toledo LF, Viegas-Péquignot E, Coutinho-Barbosa AC, Foresti F, Niveleau A, de Almeida Toledo-Filho S (1998) Localization of 5-methylcytosine in metaphase chromosomes of diploid and triploid pacu fish, Piaractus mesopotamicus (Pisces, Characiformes). Cytogenet Cell Genet 83:21-24. doi: 10.1159/000015158

Bella JL, García de la Vega C, López-Fernández C, Gosálvez J (1986) Changes in acridine orange binding and its use in the characterisation of heterochromatic regions. Heredity 57:79-83. doi: $10.1038 /$ hdy. 1986.90

Boán F, Viñas A, Rodríguez JM, Sánchez L, Gómez-Márquez J (1996) A new EcoRI family of satellite DNA in lampreys. FEBS Lett 394:187-190. doi: 10.1016/0014-5793(96)00947-7

Bracht JR (2014) Beyond transcriptional silencing: Is methylcytosine a widely conserved eukaryotic DNA elimination mechanism? Bioessays 36:346-352. doi: 10.1002/bies.201300123

Bracht JR, Perlman DH, Landweber LF (2012) Cytosine methylation and hydroxymethylation mark DNA for elimination in Oxytricha trifallax. Genome Biol 13:R99. doi: 10.1186/gb-2012-1310-r99

Caputo V, Giovannotti M, Cerioni PN, Splendiani A, Tagliavini J, Olmo E (2011) Chromosomal study of a lamprey (Lampetra zanandreai Vladykov, 1955) (Petromyzonida: Petromyzontiformes): conventional and FISH analysis. Chromosome Res 19:481-491. doi: 10.1007/s10577-011-9197-4

Chen ZJ (2007) Genetic and Epigenetic Mechanisms for Gene Expression and Phenotypic Variation in Plant Polyploids. Annu Rev Plant Biol 58:377-406. doi: 10.1146/annurev.arplant.58.032806.103835 
Dricu E (2012) Methylation: From DNA, RNA and Histones to Diseases and Treatment. InTech. Rijeka, Croatia. doi: 10.5772/2932

Feng S, Jacobsen SE, Reik W (2010) Epigenetic reprogramming in plant and animal development. Science 330:622-627. doi: 10.1126/science.1190614

Fujiwara A, Nishida-Umehara C, Sakamoto T, Okamoto N, Nakayama I, Abe S (2001) Improved fish lymphocyte culture for chromosome preparation. Genetica 111:77-89. doi: 10.1023/A:1013788626712

Grishanin A (2014) Chromatin diminution in Copepoda (Crustacea): pattern, biological role and evolutionary aspects. Comp Cytogenet 8:1-10. doi: 10.3897/CompCytogen.v8i1.5913

Jaenisch R, Bird A (2003) Epigenetic regulation of gene expression: how the genome integrates intrinsic and environmental signals. Nat Genet 33:245-254. doi: 10.1038/ng1089

Jones PA (2012) Functions of DNA methylation: islands, start sites, gene bodies and beyond. Nat Rev Genet 13:484-492. doi: 10.1038/nrg3230

Kirtiklis L, Boron A, Porycka K (2005) Chromosome banding patterns of the gudgeon, Gobio gobio (Actinopterygii, Cyprinidae). Acta Ichthyol Piscat 35:119-123.

Kloc M, Zagrodzinska B (2001) Chromatin elimination--an oddity or a common mechanism in differentiation and development? Differentiation 68:84-91. doi: 10.1046/j.14320436.2001.680202.x

Laurent L, Wong E, Li G et al. (2010) Dynamic changes in the human methylome during differentiation. Genome Res 20:320-31. doi: 10.1101/gr.101907.109

Matzke MA, Mette MF, Matzke AJM (2000) Transgene silencing by the host genome defense: implications for the evolution of epigenetic control mechanisms in plants and vertebrates. Plant Mol Biol 43:401-415. doi: 10.1023/A:1006484806925

Müller F, Tobler H (2000) Chromatin diminution in the parasitic nematodes Ascaris suum and Parascaris univalens. Int J Parasitol 30:391-399. doi: 10.1016/S0020-7519(99)00199-X

Nakai Y, Kubota S, Kohno S (1991) Chromatin diminution and chromosome elimination in four Japanese hagfish species. Cytogenet Cell Genet 56:196-198. doi: 10.1159/000133087 
Pérez-García C, Guerra-Varela J, Morán P, Pasantes JJ (2010) Chromosomal mapping of rRNA genes, core histone genes and telomeric sequences in Brachidontes puniceus and Brachidontes rodriguezi (Bivalvia, Mytilidae). BMC Genet 11:109. doi: 10.1186/1471-215611-109

Potter IC, Robinson ES (1981) New developments in vertebrate cytotaxonomy V. Cytotaxonomy of lampreys. Genetica 56:149-151. doi: 10.1007/BF00055414

Potter IC, Rothwell B (1970) The mitotic chromosomes of the lamprey, Petromyzon marinus L. Experientia 26:429-430. doi: 10.1007/BF01896930

Reik W, Dean W, Walter J (2001) Epigenetic reprogramming in mammalian development. Science 293:1089-1093. doi: 10.1126/science.1063443

Rozen S, Skaletsky H (2000) Primer3 on the WWW for general users and for biologist programmers. Methods Mol Biol 132:365-386. doi: 10.1385/1-59259-192-2:365

Smith JJ, Antonacci F, Eichler EE, Amemiya CT (2009) Programmed loss of millions of base pairs from a vertebrate genome. PNAS 106:11212-11217. doi: 10.1073/pnas.0902358106

Smith JJ, Kuraku S, Holt C et al. (2013) Sequencing of the sea lamprey (Petromyzon marinus) genome provides insights into vertebrate evolution. Nat Gent 45:415-421. doi: $10.1038 /$ ng. 2568

Smith JJ, Stuart AB, Sauka-Spengler T, Clifton SW, Amemiya CT (2010) Development and analysis of a germline BAC resource for the sea lamprey, a vertebrate that undergoes substantial chromatin diminution. Chromosoma 119:381-389. doi: 10.1007/s00412-010-0263-z

Sumner AT (1972) A simple technique for demonstrating centromeric heterochromatin. Exp Cell Res 75:304-306. doi: 10.1016/0014-4827(72)90558-7

Suzuki A, Ikeda Y, Nakayama K, Matsui F (1999) Chromosomes and Ag-NORs of three species of Lampetra (Petromyzontiformes). Chromosome Res 3:150.

Suzuki MM, Bird A (2008) DNA methylation landscapes: provocative insights from epigenomics. Nat Rev Genet 9:465-476. doi: 10.1038/nrg2341 
418 Takezaki N, Figueroa F, Zaleska-Rutczynska Z, Klein J (2003) Molecular phylogeny of early vertebrates: monophyly of the agnathans as revealed by sequences of 35 genes. Mol Biol Evol 20:287-292. doi: 10.1093/molbev/msg040

421 Vilgalys R (2005) Conserved primer sequences for PCR amplification and sequencing from nuclear ribosomal RNA. Duke University, USA. http://biology.duke.edu/fungi/mycolab/primers.htm. Accessed 10 July 2014

424 Völker M, Ráb P, Kullmann H (2005) Karyotype differentiation in Chromaphyosemion killifishes (Cyprinodontiformes, Nothobranchiidae). I: Chromosome banding patterns of C. alpha, $C$. kouamense and C. lugens. Genetica 125:33-41. doi: 10.1007/s10709-005-4267-1 
Fig. 1 Sequential DAPI/CMA/PI staining, C-banding and FISH using EcoRI sat and rpt200 probes on the chromosomes of Petromyzon marinus. Combined DAPI/CMA staining shows CMA bright bands at one of the ends of every meiotic bivalent $(\mathbf{a}, \mathbf{b})$ and mitotic chromosome $(\mathbf{g}, \mathbf{h})$. These CMA+ bands are also DAPI- $(\mathbf{c}, \mathbf{i})$, coincident with $\mathrm{C}$-positive bands $(\mathbf{d}, \mathbf{j})$ and showing bright fluorescence after PI staining (e, k). Fainter $\mathrm{C}$-bands are also located in some $\mathrm{CMA}^{0}$ regions (arrowheads $\mathbf{d}, \mathbf{j}$ ). DAPI staining also shows variations in fluorescence intensity along the chromosomes, some remarked with arrowheads (c, i). EcoRI satellite signals (SAT) appear on DAPI+ regions (arrowheads in $\mathbf{e}, \mathbf{k}$ ) close to the $\mathrm{CMA}+\mathrm{PI}+$ bands. The number of $r p t 200$ signals decreases from meiotic prophase to mitotic metaphase (asterisks in $\mathbf{f}, \mathbf{l}$ ). Double-color FISH using EcoRI satDNA (SAT) and rpt200 probes show that the signals for each probe appear on different chromosomes (f, I). Scale bars, $10 \mu \mathrm{m}$

Fig. 2 Immunolocation of 5-methylcytosine (5-mC) on meiotic bivalents and mitotic chromosomes of Petromyzon marinus counterstained with DAPI and PI. Strong 5-mC signals appear on every single $\mathrm{CMA}+/ \mathrm{PI}+$ region on meiotic bivalents (arrows in $\mathbf{b}$ ) and are also present at some intercalary DAPI faint bands (arrowheads in $\mathbf{a}, \mathbf{b}$ ). 5-mC signals are also coincident with $\mathrm{CMA}+\mathrm{PI}+$ regions on mitotic chromosomes but the signals are both less intense and not present in all chromosomes

Fig. 3 Chromosomal location of major rDNA, rpt200 and 5' Germ1 in Petromyzon marinus. 28s rDNA probe yielded signals on nine different bivalents (arrows in a) but in only a single mitotic chromosome pair (arrows in d). rpt200 signals appear on DAPI-dull regions of some bivalents, most of them close to the bright CMA/PI regions (b), and in a lower number on mitotic chromosomes (e). Double FISH experiments using $28 \mathrm{~S}$ rDNA and rpt200 probes labeled differently revealed that all $28 \mathrm{~S}$ rDNA signals coincide with rpt200 signals, but there are rpt200 signals devoid of $28 \mathrm{~S}$ rDNA signals, too (arrowheads c, f). Double-color FISH experiments using 5' Germ 1 and $28 \mathrm{~S}$ rDNA probes always show overlapping signals in both meiotic and mitotic chromosomes (i, I). Single FISH images for $5^{\prime}$ Germ $1(\mathbf{g}, \mathbf{j})$ and $28 \mathrm{~s}$ rDNA (h, k). Scale bars, $10 \mu \mathrm{m}$

Fig. 4 Schematic representation of the chromosomal markers on meiotic and mitotic chromosomes of Petromyzon marinus. Germ1 is eliminated in 8 chromosome pairs (a) and kept in one (b). 


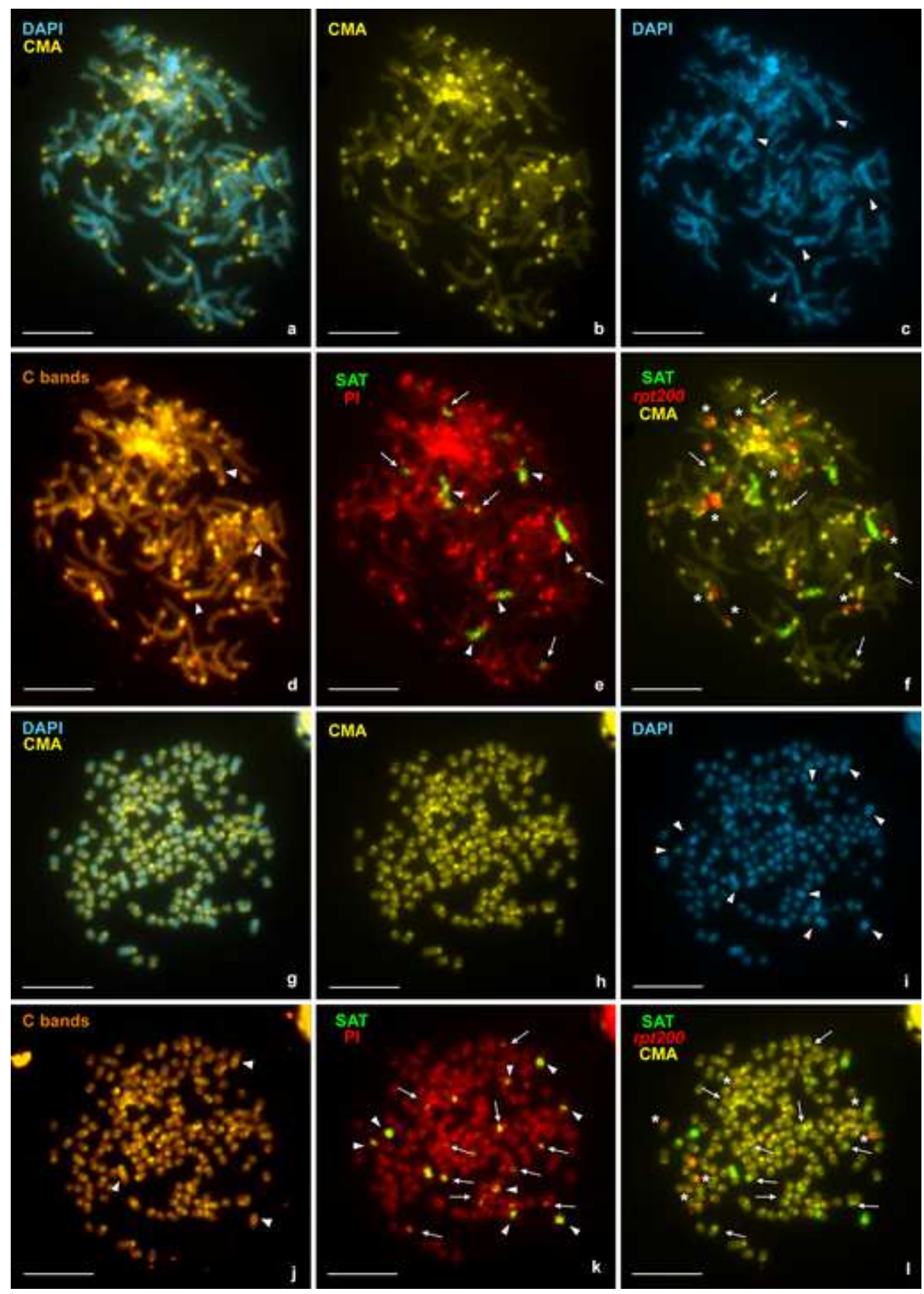




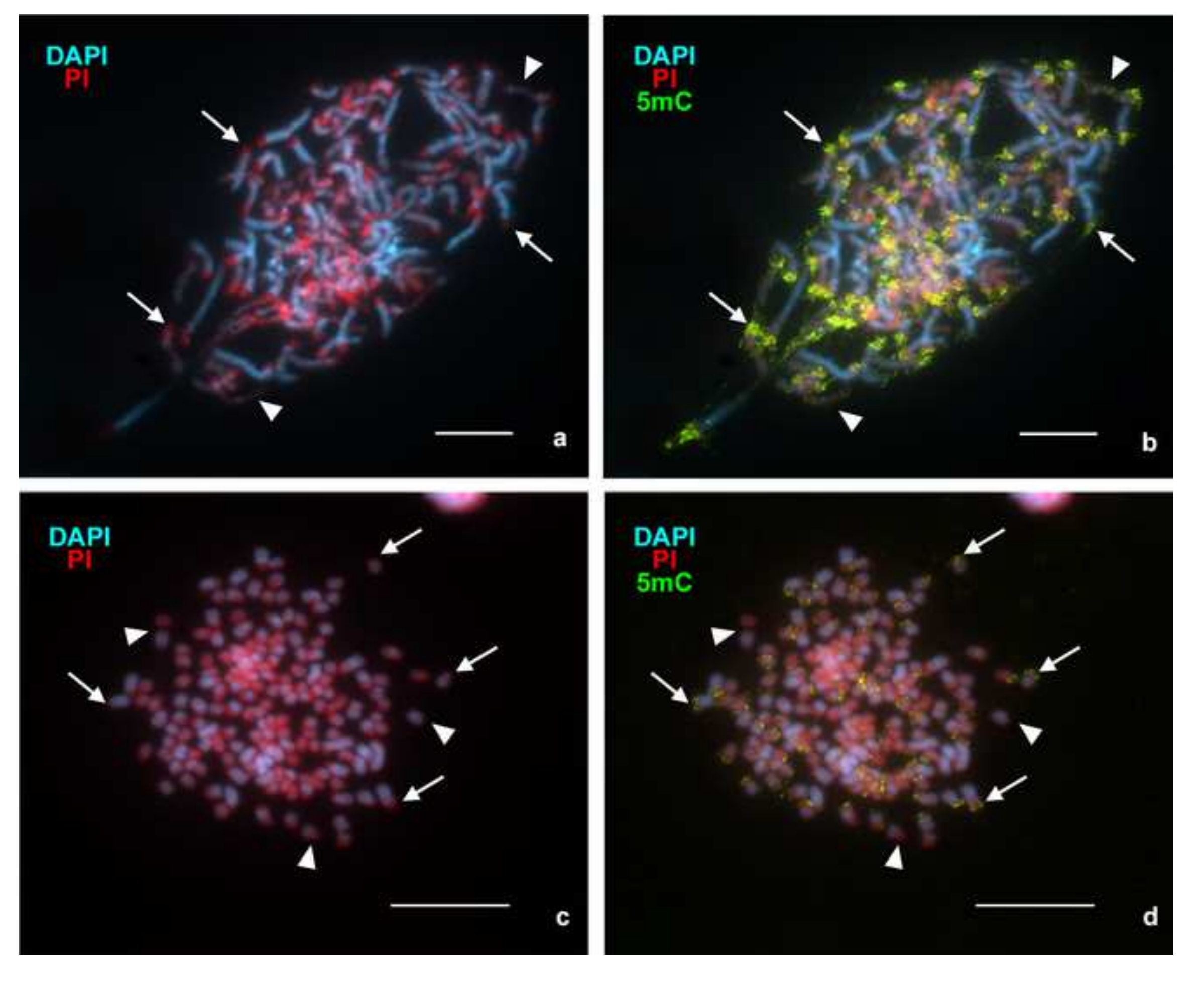




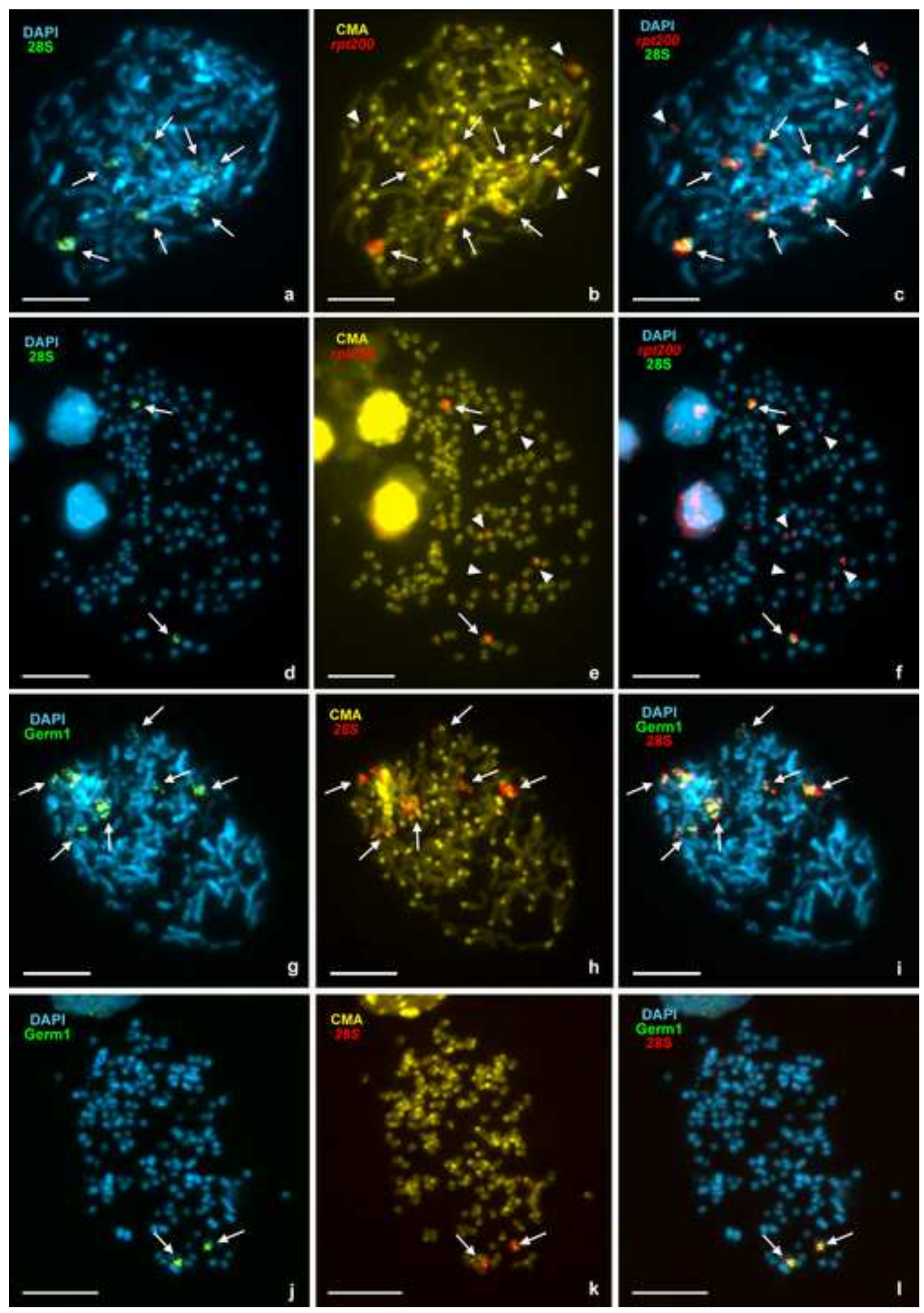




\section{Germ line}

Chromosomes in meiotic prophase I

Somatic line

Chromosomes in mitotic metaphase
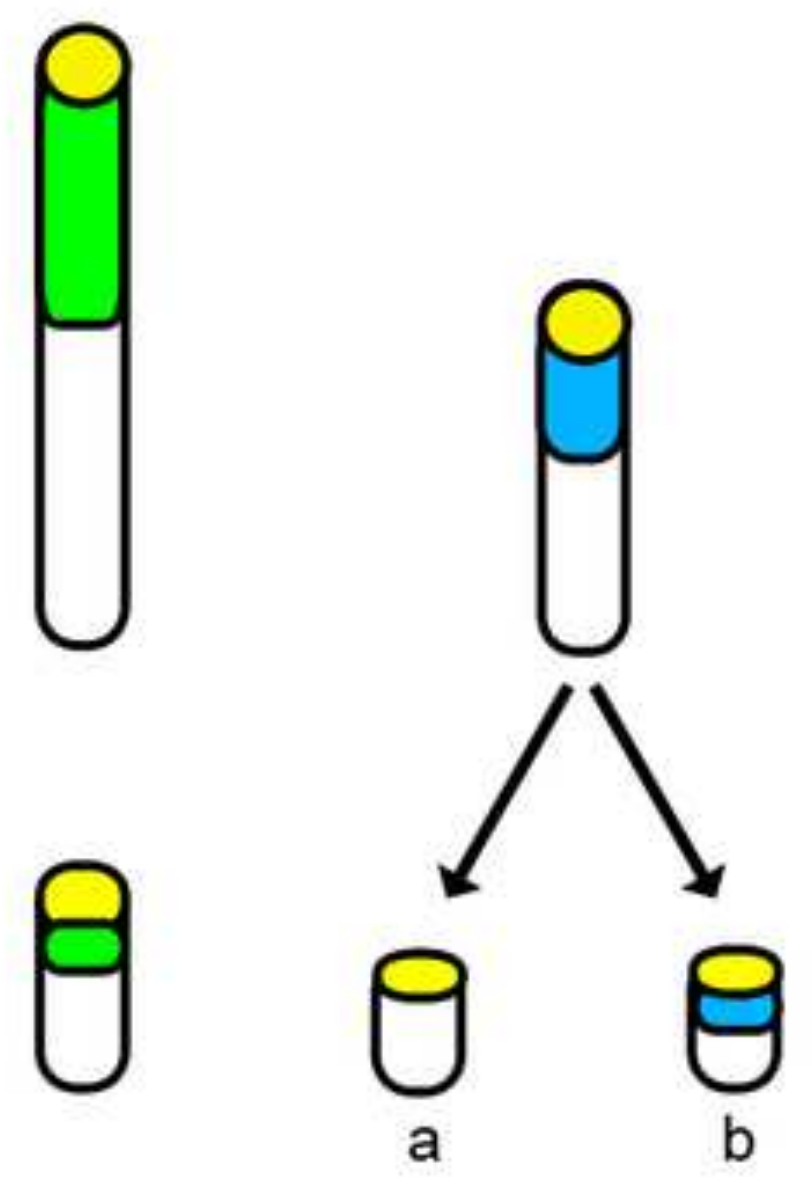
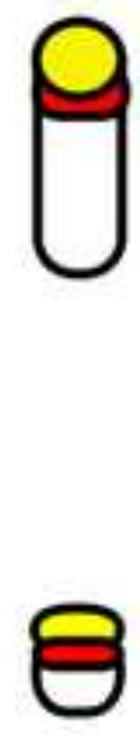

$\square \mathrm{CMA}^{+} / \mathrm{PI}^{+} / \mathrm{DAPI} / \mathrm{C}$-bands/5-mC

$\square$ Germ1/rpt200/major rDNA

$\square$ EcoRI satDNA

$\square$ rpt200 
Table 1

Click here to download Table: Covelo-Soto et al 2014 Table 1.docx

Table 1 Combinations of primers used in the PCR amplification

\begin{tabular}{|c|c|c|}
\hline Region & Primers & Primer sequence $\left(5^{\prime} \rightarrow 3^{\prime}\right)$ \\
\hline \multirow{2}{*}{$28 S$ rDNA } & $L R 10 R$ & GACCCTGTTGAGCTTGA \\
\hline & $L R 12$ & GACTTAGAGGCGTTCAG \\
\hline \multirow{2}{*}{ rpt200 } & $r p t F$ & GAAATGCATGTGCACTCAAAA \\
\hline & $r p t R$ & ATGGGGTTGAATGCTTTTTTG \\
\hline \multirow{2}{*}{ EcoRI satDNA } & satF & TCTCCCCGTCTGGACGAGAGGAA \\
\hline & satR & GACCCAAACCGAGAGGATTCTGGG \\
\hline \multirow{2}{*}{ 5' Germ1 } & $5^{\prime}$ germF & ACGTATCGATGAGGGAGCAC \\
\hline & $5^{\prime}$ germR & TGTTCCACCGTCAAAAGTGA \\
\hline
\end{tabular}


Table 2 Parameters of the PCR amplification

\begin{tabular}{|c|c|c|c|c|c|}
\hline \multirow{2}{*}{ Probe } & \multirow{2}{*}{$\begin{array}{c}\text { Initial } \\
\text { denaturation }\end{array}$} & \multicolumn{3}{|c|}{30 cycles } & \multirow{2}{*}{$\begin{array}{c}\text { Final } \\
\text { elongation }\end{array}$} \\
\hline & & Denaturation & Hybridization & Elongation & \\
\hline $28 S$ rDNA & $95 \stackrel{\circ}{ } \mathrm{C}, 5 \mathrm{~min}$ & $95 \stackrel{\circ}{ } \mathrm{C}, 20 \mathrm{~s}$ & $48 \stackrel{\circ}{ } \mathrm{C}, 20 \mathrm{~s}$ & $72 \stackrel{\circ}{ } \mathrm{C}, 20 \mathrm{~s}$ & $72 \stackrel{\circ}{\circ}, 7 \mathrm{~min}$ \\
\hline rpt200 & $94 \stackrel{\circ}{ } \mathrm{C}, 5 \mathrm{~min}$ & $94 \stackrel{\circ}{C}, 45 \mathrm{~s}$ & $59 \stackrel{\circ}{ }, 45 \mathrm{~s}$ & $72 \mathrm{o} C, 30 \mathrm{~s}$ & $72 \stackrel{\circ}{\circ}, 7 \mathrm{~min}$ \\
\hline EcoRI satDNA & $95 \stackrel{\circ}{ } \mathrm{C}, 5 \mathrm{~min}$ & $95 \stackrel{\circ}{ } \mathrm{C}, 20 \mathrm{~s}$ & $57 \stackrel{\circ}{C}, 20 \mathrm{~s}$ & $72 \stackrel{\circ}{ } \mathrm{C}, 20 \mathrm{~s}$ & $72 \stackrel{\circ}{\circ}, 7 \mathrm{~min}$ \\
\hline 5' Germ1 & $95 \stackrel{\circ}{\circ}, 5 \mathrm{~min}$ & $95 \stackrel{\circ}{\circ}, 8 \mathrm{~s}$ & $60 \stackrel{\circ}{ }, \quad 8 \mathrm{~s}$ & $72 \stackrel{\circ}{ } \mathrm{C}, \quad 8 \mathrm{~s}$ & $72 \stackrel{\circ}{ } \mathrm{C}, 7 \mathrm{~min}$ \\
\hline
\end{tabular}


Click here to download Table: Covelo-Soto et al 2014 Table 3.docx

Table 3 Number of 5-mC, EcoRI satDNA, rpt200 and 28 S signals in meiotic prophase I and mitotic metaphase plates of sea lamprey. The number of expected mitotic signals are also given

\begin{tabular}{|c|c|c|c|c|c|c|c|c|c|c|c|c|}
\hline & \multicolumn{3}{|c|}{$5-\mathrm{mC}$} & \multicolumn{3}{|c|}{ EcoRI satDNA } & \multicolumn{3}{|c|}{ rpt200 } & \multicolumn{3}{|c|}{$28 S$} \\
\hline & \multirow{2}{*}{ Meiotic plates } & \multicolumn{2}{|c|}{ Mitotic plates } & \multirow{2}{*}{ Meiotic plates } & \multicolumn{2}{|c|}{ Mitotic plates } & \multirow{2}{*}{ Meiotic plates } & \multicolumn{2}{|c|}{ Mitotic plates } & \multirow{2}{*}{ Meiotic plates } & \multicolumn{2}{|c|}{ Mitotic plates } \\
\hline & & Observed & Expected & & Observed & Expected & & Observed & Expected & & Observed & Expected \\
\hline Mean & $76.75 \pm 0.87$ & $69.94 \pm 1.00$ & $153.50 \pm 1.74$ & $16.22 \pm 0.20$ & $31.31 \pm 0.26$ & $32.44 \pm 0.41$ & $19.94 \pm 0.12$ & $28.16 \pm 0.30$ & $39.88 \pm 0.24$ & $10.91 \pm 0.16$ & $2.00 \pm 0.00$ & $21.81 \pm 0.32$ \\
\hline Median & 77.50 & 65.00 & 155.00 & 16.00 & 31.00 & 32.00 & 20.00 & 29.00 & 40.00 & 11.00 & 2.00 & 22.00 \\
\hline Mode & 71 & 64 & 142 & 16 & 32 & 32 & 20 & 27 & 40 & 10 & 2 & 20 \\
\hline Range & $70-85$ & $57-76$ & $140-170$ & $14-19$ & $28-34$ & $28-38$ & $19-21$ & $25-30$ & $38-42$ & $10-12$ & 2 & $20-24$ \\
\hline
\end{tabular}


Supplemental File
Click here to download Supplemental Materials: Table 1 Supplementary file.pdf

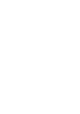

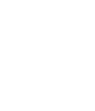

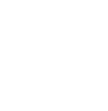
-

(

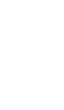

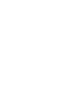

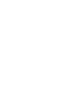

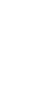

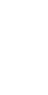

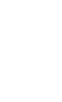
. (n) (n) (n) (n) (n) (n) (n) ( .

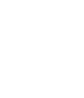
. . . . . . .

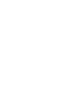

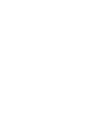
.

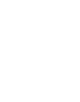

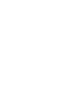

